\title{
A geometric criterion for compactness of invariant subspaces
}

\author{
Leszek Skrzypczak and Cyril Tintarev
}

\begin{abstract}
Let $M$ be a non-compact homogeneous Riemannian manifold, and let $\Omega$ be a compact subgroup of isometries of $M$. We show, under general conditions, that the $\Omega$-invariant subspace $A_{\Omega}$ of a normed vector space $A \hookrightarrow L^{q}(M)$ is compactly embedded into $L^{q}(M)$ if and only if the group $\Omega$ has no orbits with a uniformly bounded diameter in a neighborhood of infinity.
\end{abstract}

Mathematics Subject Classification (2010). Primary 46B50; Secondary 46E35, 46N20.

Keywords. Convergence, Compactness, Concentration.

1. Introduction. The problem studied in this paper has its origin in the compactness result of Strauss [14]: while the Sobolev embedding $W^{1,2}\left(\mathbb{R}^{N}\right) \hookrightarrow$ $L^{q}\left(\mathbb{R}^{N}\right), q \in\left(2, \frac{2 N}{N-2}\right), N>2$, is not compact, the subspace of radially symmetric functions of $W^{1,2}\left(\mathbb{R}^{N}\right)$ is embedded into $L^{q}\left(\mathbb{R}^{N}\right)$ compactly. Numerous generalizations of this result have been obtained for various domain and target spaces, as well as for different symmetry conditions, cf. $[2,8,9,11,12]$. In particular, the Sobolev spaces on Riemannian manifolds were studied by Hebey, Vaugon [5,6], and one of the authors [13]. A more abstract approach was developed by the second author and his collaborators $[3,10,17]$

The purpose of this paper is to identify a general geometric condition that is necessary and sufficient for such a compactness phenomenon to occur in a setting when the original embedding is inherently non-compact. We consider an embedding of an abstract normed space $A$ into the Lebesgue space of a non-compact complete Riemannian manifold $M$. All integrals in this paper are taken with respect to the Riemannian measure on $M$.

Throughout the paper we will use the following set of assumptions.

(A) Let $1<q<\infty$, and let $A \hookrightarrow L^{q}(M)$ be a normed vector space. Let $G$ be a subgroup of the group of isometries $I(M)$ of $M$ that acts transitively 
on $M$, and assume that the norm of $A$ is invariant with respect to the actions of $G$, i.e. that $u \circ \eta \in A$ if $u \in A, \eta \in G$, and

$$
\|u \circ \eta\|_{A}=\|u\|_{A} \text {. }
$$

The non-compactness of this embedding is assured by the following elementary statement.

Proposition 1.1. Assume (A). If A contains a function with compact support, then the embedding $A \hookrightarrow L^{q}(M)$ is not compact.

Proof. Let $u \in A$ be a function supported on a compact set $B$. Since $M$ is not compact, it contains infinitely many disjoint geodesic balls of radius $R$. Since $G$ acts transitively, there exists an infinite set of elements of constant $A$-norm of the form $u \circ \eta, \eta \in G$, with disjoint supports, and thus equidistant in $L^{q}(M)$.

We prove in this paper that, in a general setting, compactness of a symmetric subspace of $A$ in $L^{q}$ is equivalent to the following geometric condition.

Definition 1.2. We say that a continuous action of a group $\Omega$ on a complete Riemannian manifold $M$ is coercive if for every $t>0$, the set

$$
O_{t}=\{x \in M: \operatorname{diam} \Omega x \leq t\}
$$

is bounded.

The planar rotation action of $S O(2)$ on $\mathbb{R}^{3}$ is not coercive because for every $t>0$ the set $O_{t}$ is a circular cylinder. The action of $S O(3)$ on $\mathbb{R}^{3}$ is coercive because for every $t>0$ the set $O_{t}$ is the closed ball of radius $t / 2$.

In order to formulate our main result, we need to define an important property of the space $A$.

An continuous embedding of a normed vector space $A$ into a reflexive Banach space $X$ is called weakly cocompact relative to a bounded set $D$ of bounded linear operators acting on $A$ if any bounded sequence $u_{k} \in A$ such that $\forall g_{k} \in D, g_{k} u_{k} \rightarrow 0$ in $X$ (which we write as $u_{k} \stackrel{D}{\rightarrow} 0$ ) converges to zero in the norm of $X$. Here $\rightarrow$ denotes the weak convergence in $X$. In what follows we will also use the notation $u_{k} \stackrel{\text { a.e. }}{\rightarrow} u$ in $L^{q}(M)$ to indicate that the sequence $u_{k}$ converges weakly in $L^{q}$ and almost everywhere on $M$.

Weak cocompactness of an embedding is a property related to, but generally weaker than compactness. If $X^{*}$ is dense in $A^{*}$, then weak cocompactness is trivially equivalent to cocompactness as defined in earlier work, e.g. [3].

If the space $A$ and the group $G$ satisfy the assumption (A), then we can consider the weak cocompactness relative to the action of $G$, i.e. with respect to the set of isometries of $A$ defined by the elements of the group $G$. If the group $G$ is trivial, then cocompactness is trivially equivalent to compactness.

Cocompactness plays a crucial role in the existence of extrema in noncompact minimization problems, and is an underlying phenomenon of the concentration compactness principle introduced by Lions (e.g. [9]). In Section 4 we give a list of cocompact embeddings into $L^{q}$, which of course includes Sobolev embeddings on homogeneous manifolds. 
Theorem 1.3. Let $M$ be a complete connected noncompact Riemannian manifold. Let $A \hookrightarrow L^{q}(M), q \in(1, \infty), A$ and $G$ be, respectively, a normed vector space and a subgroup of $I(M)$ that satisfy assumptions (A). Assume that the embedding $A \hookrightarrow L^{q}(M)$ is weakly cocompact relative to the action of $G$. Suppose also that every sequence bounded in $A$ has a subsequence which converges a.e. Let $\Omega$ be a compact connected subgroup of $I(M)$, and let $A_{\Omega}$ denote the subspace of $\Omega$-invariant functions in $A$. Then if $\Omega$ is coercive, the embedding of $A_{\Omega}$ into $L^{q}(M)$ is compact.

Conversely, if $A$ contains a compactly supported function whose integral is not zero, then the compactness of the embedding $A_{\Omega} \hookrightarrow L^{q}(M)$ implies that $\Omega$ is coercive.

The conditions of the theorem are obviously satisfied by Sobolev spaces of $\mathbb{R}^{N}$ and radial or suitable block-radial symmetries, cf. Section 3 .

The paper is organized as follows. In Section 2 we prove the main theorem. In Section 3 we give a general geometric example of a coercive symmetry group, and in Section 4 we present examples of spaces $A$ satisfying the conditions of the theorem. For convenience of the reader, we recall a covering lemma and an iterated Brezis-Lieb lemma in the last part of the paper.

2. Proof of main theorem. We say that a sequence of points $x_{n} \in M$ converges to infinity if for any compact set $K \subset M$ there is an integer $N$ such that for all $n \geq N, x_{n} \notin K$.

Lemma 2.1. Assume that the group $\Omega$ is connected and acts coercively on $M$. Then for any sequence $x_{k} \rightarrow \infty$, there exists a sequence of elements $\omega_{k}^{(1)}, \ldots, \omega_{k}^{(k)} \in \Omega$ such that a renumbered subsequence of $x_{k}$ satisfies

$$
d\left(\omega_{k}^{(m)} x_{k}, \omega_{k}^{(n)} x_{k}\right) \rightarrow \infty \text { whenever } m \neq n .
$$

Proof. Note that each orbit of $\Omega$ is a compact connected manifold. Let $r>0$ and $x \in M$. By Lemma 5.1 there exist $\omega_{1 ; r, x}, \ldots, \omega_{m(r, x) ; r, x} \in \Omega$ such that $\Omega x$ is covered by the balls $B_{2 r}\left(\omega_{1 ; r, x} x\right), \ldots, B_{2 r}\left(\omega_{m(r, x) ; r, x} x\right)$, while the balls $B_{r}\left(\omega_{1 ; r, x} x\right), \ldots, B_{r}\left(\omega_{m(r, x) ; r, x} x\right)$ are pairwise disjoint. Let $x_{k} \rightarrow \infty$. Assume that there exists a $C(r)$, such that $m\left(r, x_{k}\right) \leq C(r)$. Then diam $\Omega x_{k} \leq 4 r C(r)$. In other words, $x_{k} \in O_{4 r C(r)}$. This implies, since $\Omega$ is coercive, that the sequence $x_{k}$ is bounded, which is a contradiction. Consequently, $m\left(r, x_{k}\right) \rightarrow \infty$ for every $r$. Select now $x_{j_{k}}$ large enough so that $m\left(k, x_{j_{k}}\right) \geq k$.

Lemma 2.2. Under the assumptions of Lemma 2.1, for every $J \in \mathbb{N}$ there exists a renumbered subsequence of $x_{k}$ such that the corresponding renumbered sequences $\omega_{k}^{(1)}, \ldots, \omega_{k}^{(J)}$, given by Lemma 2.1, converge in $\Omega$ as $k \rightarrow \infty$ to the respective limits $\omega^{(1)}, \ldots, \omega^{(J)}$.

The assertion follows from an elementary induction argument using the diagonal argument. We recall that the sequence $\eta_{k} \in I(M)$ is called discrete if there exists a point $x \in M$ such that $\eta_{k}(x) \rightarrow \infty$. Note that if the action of the group $G$ is transitive and the property holds for some $x$, then it holds 
for every $x$ in $M$. Moreover if $\eta_{k}$ is discrete, then also $\eta_{k}^{-1}$ is discrete. We are ready to prove Theorem 1.3 .

Proof. Sufficiency: Let $\left\{u_{k}\right\}_{k \in \mathbb{N}}$ be an arbitrary bounded sequence in $A_{\Omega}$. We may assume without loss of generality that $u_{k} \stackrel{\text { a.e. }}{\longrightarrow} u$ in $L^{q}(M)$. The embedding is weakly cocompact, therefore if $u_{k}$ has no subsequence convergent in $L^{q}(M)$, then there is a sequence $\left\{\eta_{k}\right\}_{k \in \mathbb{N}}$ in $\mathrm{G}$ such that, on a renamed subsequence, we have

$$
\left(u_{k}-u\right) \circ \eta_{k} \stackrel{-1}{\stackrel{\text { a.e. }}{\longrightarrow}} y \neq 0 .
$$

The sequence $\eta_{k}{ }^{-1}$ is necessarily discrete since if it is not discrete then $\eta_{k}{ }^{-1} x$ converges for a renumbered subsequence and some $x \in M$. But $\eta_{k}{ }^{-1}$ is a sequence of isometries of connected metric space, therefore convergence at one point implies, for a renumbered subsequence of $\eta_{k}{ }^{-1}$, convergence at any point of $M$. In consequence the sequence $\eta_{k} \rightarrow \eta \in G$ in the compact-open topology of $I(M)$. Then for any $v \in L^{q^{\prime}}$,

$$
\left\langle v,\left(u_{k}-u\right) \circ \eta_{k}^{-1}\right\rangle=\left\langle v \circ \eta_{k}, u_{k}-u\right\rangle \rightarrow 0
$$

since $v \circ \eta_{k} \rightarrow v \circ \eta$ in $L^{q^{\prime}}$. But this contradicts the assumption that $y \neq 0$.

Let $\omega_{k} \rightarrow \omega \in \Omega$. Then it follows from (2.1) that

$$
\left(u_{k}-u\right) \circ \omega_{k} \circ \eta_{k}^{-1} \circ \omega_{k}^{-1} \stackrel{\text { a.e. }}{\longrightarrow} y \circ \omega^{-1} .
$$

Fix $J \in \mathbb{N}$. Let $a \in M$ be an arbitrary point, and let $x_{k}=\eta_{k} a$. By Lemmas 2.1 and 2.2 , there exist sequences $\omega_{k}^{(1)}, \ldots, \omega_{k}^{(J)}$ that converge in $\Omega$ as $k \rightarrow \infty$ to, respectively, $\omega^{(1)}, \ldots, \omega^{(J)}$, and such that $d\left(\omega_{k}^{(m)} x_{k}, \omega_{k}^{(n)} x_{k}\right) \rightarrow \infty$ whenever $m<n \leq J$. Therefore the sequence $\eta_{k}^{-1}\left(\omega_{k}^{(m)}\right)^{-1} \omega_{k}^{(n)} \eta_{k}$ is discrete, and, consequently, the sequence

$$
\left(\omega_{k}^{(m)} \circ \eta_{k}^{-1} \circ\left(\omega_{k}^{(m)}\right)^{-1}\right) \circ\left(\omega_{k}^{(n)} \circ \eta_{k}^{-1} \circ\left(\omega_{k}^{(n)}\right)^{-1}\right)^{-1}
$$

is also discrete.

Since $u \circ \eta_{k} \stackrel{\text { a.e. }}{\rightarrow} 0$ in $L^{q}(M)$ for any discrete sequence $\eta_{k} \in G$ (it is immediate for $u$ with compact support and extends to the whole $L^{q}(M)$ by density), the iterated Brezis-Lieb lemma, i.e. Lemma 5.1, applies and yields

$$
\liminf \int_{M}\left|u_{k}\right|^{q} d \mu \geq J \int|y|^{q} d \mu .
$$

For a $J$ sufficiently large (and a corresponding subsequence), we obtain a contradiction which proves the compactness of the embedding.

Necessity: If $\Omega$ is not coercive, there exists $R>0$ and a sequence $x_{k} \rightarrow \infty$ such that $\Omega x_{k} \subset B_{R}\left(x_{k}\right)$. By the assumption there exists a function $\psi$ with $\int_{M} \psi>0$ supported in a ball $B_{r}(y)$ for some $y \in M$ and $r>0$. Let us replace $x_{k}$ with a renumbered subsequence such that the distance between any two terms in the sequence will be greater than $2(R+r)$. Let $\eta_{k} \in G$ be such that 
$\eta_{k} x_{k}=y$, and define

$$
\psi_{k}=\int_{\Omega} \psi \circ \eta_{k} \circ \omega \mathrm{d} \omega
$$

where the Haar measure of $\Omega$ is normalized to the value 1 . By the Young inequality,

$$
\left\|\psi_{k}\right\|_{A} \leq \int_{\Omega}\left\|\psi \circ \eta_{k} \circ \omega\right\|_{A} \mathrm{~d} \omega=\int_{\Omega}\|\psi\|_{A} \mathrm{~d} \omega=\|\psi\|_{A} .
$$

Note that the supports of the functions $\psi_{k}$ are disjoint, and therefore

$$
\left\|\psi_{m}-\psi_{n}\right\|_{L^{q}}=\left\|\psi_{m}\right\|_{L^{q}}+\left\|\psi_{n}\right\|_{L^{q}} \geq 2 \inf _{k}\left\|\psi_{k}\right\|_{L^{q}} .
$$

Furthermore,

$$
\mu\left(B_{R+r}\right)^{1-1 / q}\left\|\psi_{k}\right\|_{L^{q}} \geq \int_{M} \psi_{k}=\int_{\Omega} \int_{M} \psi \circ \eta_{k} \circ \omega \mathrm{d} \mu \mathrm{d} \omega=\int_{M} \psi \mathrm{d} \mu>0 .
$$

Consequently, we have a sequence, bounded in $A$ and lacking a convergent subsequence in $L^{q}$, and so the embedding $A_{\Omega} \hookrightarrow L^{q}(M)$ is not compact.

Remark 2.3. - The reasoning in the proof of necessity extends with trivial changes to embeddings into any reflexive rearrangement-invariant space that is continuously embedded into $L_{\text {loc }}^{1}(M)$.

- The theorem remains valid with only elementary adaptations of the proofs if instead of the group of isometries $I(M)$ of the manifold $M$, one takes a locally compact group $G$ of homeomorphisms acting transitively on $M$, equipped with the consistent compact-open topology. One needs to assume that the group $G$ acts transitively and equicontinuously on $M$, preserves the Riemannian measure of $M$, for some $C>0$ satisfies $\sup _{\eta \in G}$ $\|u \circ \eta\|_{A} \leq C\|u\|_{A}$. In this setting $\Omega$ is a compact coercive subgroup of $G$.

Corollary 2.4. Assume that the group $\Omega$ fixes a point $o \in M$, and let $A_{\text {rad }}$ be the subspace of $A$ consisting of all functions dependent on $r=d(o, x)$. Then under the remaining conditions of Theorem 1.3, if $\Omega$ is coercive, then the embedding of $A_{\mathrm{rad}} \hookrightarrow L^{q}(M)$ is compact.

Proof. We have $A_{\text {rad }} \hookrightarrow A_{\Omega}$.

3. Coercive groups. In a more restrictive setting, we have the following necessary and sufficient condition for the action of a subgroup $G \subset I(M)$ to be coercive.

Proposition 3.1. Let $M$ be a simply connected complete Riemannian manifold, and assume that the sectional curvature $K$ of $M$ is non-positive. Let $\Omega$ be a compact, connected subgroup of $I(M)$ that fixes some point $o \in M$. Then $\Omega$ is coercive if and only if $\Omega$ has no other fixed point but o.

Before we prove the proposition, we need a few auxiliary results. We will use the following statement, derived from [4, p. 218, Proposition 2.5]: 
Proposition 3.2. Let $M$ be a Riemannian manifold, let $o \in M$, and assume that $\exp _{o} M$ is a diffeomorphism. If $\ell$ is a curve on $T_{o} M$ and the sectional curvature of $M$ is non-positive, then the length of $\exp _{o} \ell$ on $M$ is greater or equal than the length of $\ell$.

The following statement is well known.

Lemma 3.3. Under the assumptions of Proposition 3.1, we have the following identity for all $\omega \in \Omega$ :

$$
\exp _{o} \circ d \omega=\omega \circ \exp _{o}
$$

and consequently, the map

$$
\psi_{\lambda}(x)=\exp _{o}^{-1}\left(\lambda \exp _{o}(x)\right)
$$

is a diffeomorphism between orbits on $S_{r}(o)$ and $S_{\lambda r}(o)$ for any $r, \lambda>0$.

We are ready now to prove Proposition 3.1.

Proof. Necessity: By the Hadamard-Cartan theorem, $M$ is diffeomorphic to $\mathbb{R}^{N}$ under the exponential map at any given point of $M$. Assume that for some $\rho>0$ there exists a fixed point $x_{\rho} \in S_{\rho}(o)$. Then it follows from Lemma 3.3 that for any $r>0$ the point $x_{r}=\exp _{o}\left(\frac{r}{\rho} \exp _{o}^{-1}\left(x_{\rho}\right)\right)$ is also a fixed point of $\Omega$ and $d\left(x_{r}, o\right) \rightarrow \infty$. So the group $\Omega$ is not coercive.

Sufficiency: Assume that there exists a constant $C>0$ such that for a sequence $R_{k} \rightarrow \infty$, the geodesic sphere $S_{R_{k}}(o)$ has a point $x_{k}$ such that $\operatorname{diam} \Omega x_{k} \leq C$. Now Proposition 3.2 implies

$$
\operatorname{diam}_{o}\left(\exp _{o}^{-1}\left(\Omega \cdot x_{k}\right)\right) \leq C,
$$

where the diameter diam $_{o}$ is taken with respect to the Euclidean distance on $T_{o} M$. Moreover,

$$
d \Omega \cdot \exp _{o}^{-1}\left(x_{k}\right)=\exp _{o}^{-1}\left(\Omega \cdot x_{k}\right),
$$

where $d \Omega=\left\{d_{o} \omega: \omega \in \Omega\right\}$, cf. Lemma 3.3. Therefore the set $R_{k}^{-1} \exp _{o}^{-1}\left(\Omega \cdot x_{k}\right)$ is a subset of the unit sphere in $T_{o} M$, and its diameter satisfies

$$
\operatorname{diam}_{o}\left(R_{k}^{-1} \exp _{o}^{-1}\left(\Omega \cdot x_{k}\right)\right)=\operatorname{diam}\left(R_{k}^{-1} d \Omega \cdot \exp _{o}^{-1}\left(x_{k}\right)\right) \leq \frac{C}{R_{k}} .
$$

But then the group $d \Omega$ has a fixed point belonging to the unit sphere in $T_{o} M$, so also $\Omega$ has a fixed point belonging to $S_{1}(o)$.

Example. In general, there exist coercive groups that have no fixed points, i.e. their orbits expand towards infinity and never shrink to a point. We give a simple example.

Let $M=S^{1} \times \mathbb{R}^{n}, n \geq 2$, be the Riemannian product manifold of the unit circle and the Euclidean space. Let $\Omega=S^{1} \times S O(n)$. Then $\Omega$ is a connected group of isometries acting on $M$. The action is given by the formulae $\left(e^{i \varphi}, h\right)\left(e^{i \psi}, x\right)=\left(e^{i(\varphi+\psi)}, h(x)\right), e^{i \varphi}, e^{i \psi} \in S^{1}, h \in S O(n)$, and $x \in \mathbb{R}^{n}$. Every orbit of the group $\Omega$ has a form $S^{1} \times S_{r}(0)$ with some $r \in[0, \infty)$. Therefore $\Omega$ is a compact subgroup of $I(M)$ that acts coercively on $M$ and has no fixed point. 
4. Examples. Let $M$ be as in Theorem 1.3. In particular, it may be by the hyperbolic space, $\mathbb{R}^{N}$, or a stratified nilpotent Lie group with the left or the right shift invariant Riemannian structure.

The Sobolev spaces $W^{s, p}(M), p \in(1, \infty), s \in[1, \infty)$ are cocompactly embedded into $L^{q}(M), q \in\left(p, p^{*}\right)$, where $p^{*}=\frac{p N}{N-s p}$ for $s p<N$ and $p^{*}=\infty$ for $s p \geq N$, relative to $I(M)$. The cocompactness statement originates in the work of Lieb [7]. For the case of integer $k$, we refer the reader to [17]. For fractional $s$, the result follows from the continuity of Sobolev embeddings (see Strichartz [15]), the monotonicity of the Sobolev scale with respect to $s$, and the Hölder inequality. For $M=\mathbb{R}^{N}$, and $0<s<1$, the cocompactness is verified in [3]. Thus, given the cocompactness of the embedding into $L^{q}(M)$, Theorem 1.3 implies that, for any coercive compact subgroup $\Omega$ of $I(M)$, the $\Omega$-invariant subspace of $W^{s, p}(M)$ is compactly embedded into $L^{q}$. In the case of $\mathbb{R}^{N}$, the hyperbolic space or a Carnot group, the coercivity of $\Omega$ means, by Proposition 3.1, that $\Omega$ has exactly one fixed point. In particular, in the case of $\mathbb{R}^{N}$ or $\mathbb{H}^{N}$ in the Poincaré ball coordinates, $\Omega$ may be $S O(N)$, or, more generally, consist of block-matrices

$$
\left[\begin{array}{llll}
T_{1} & 0 & \ldots & 0 \\
0 & T_{2} & \ldots & 0 \\
\ldots & \ldots & \ldots & \ldots \\
0 & \ldots & 0 & T_{J}
\end{array}\right]
$$

where $T_{j} \in S O\left(\mathbb{R}^{N_{j}}\right), \sum_{j=1}^{J} N_{j}=N, N_{j} \geq 2$.

For Besov spaces, the cocompactness of subcritical Jawerth's embeddings $B_{p, r}^{s}\left(\mathbb{R}^{N}\right) \hookrightarrow L^{q}\left(\mathbb{R}^{N}\right), q \in\left(p, p^{*}\right)$, where $p^{*}=\frac{p N}{N-s p}$ for $s p<N$ and $p^{*}=\infty$, was proved in [3]. Therefore, in this case the $\Omega$-symmetric spaces of $B_{p, r}^{s}\left(\mathbb{R}^{N}\right)$ are cocompactly embedded into corresponding $L^{q}$, if and only if $\Omega$ is coercive. When $\Omega$ is a group of block-symmetric matrices, this result was proved in [12], Corollary 3.

Let now $M$ and $\Omega$ be as in Example 3. Then the $\Omega$-invariant subspace $W_{\Omega}^{s, p}(M)$ is compactly embedded into $L^{q}(M, \mu)$ for any $p<q<p^{*}$, where $p^{*}=\frac{p}{1-s p}$ if $s p<1$ and $p^{*}=\infty$ otherwise. This is immediate from the fact that the functions in $W_{\Omega}^{s, p}(M)$ are in fact functions of one variable. This example, however, bears on the more general situation when the dimension of the orbit is involved in decreasing the effective local dimension of the manifold which may lead to a corresponding increase of the value of the critical Sobolev exponent. See more details in $[6,13]$.

We would like also to mention the cocompactness of the Strichartz embeddings into $L^{q}$ for the nonlinear Schrödinger equation by Terence Tao [16] (Tao calls the cocompactness property "inverse embedding"). Similarly to the critical Sobolev inequality, the embedding of the radial subspace is not compact, as the loss of compactness may occur due to rescalings.

Acknowledgements. The authors would like to thank the referee for helpful remarks. 
Open Access. This article is distributed under the terms of the Creative Commons Attribution License which permits any use, distribution, and reproduction in any medium, provided the original author(s) and the source are credited.

\section{Appendix.}

A covering lemma. The following lemma in different versions occurs many times in literature, we quote a particular case of Lemma A.1 from [17].

Lemma 5.1. Let $X$ be a compact subset of a complete Riemannian manifold $M$, and let $r>0$. There exists a finite covering of $X$ by open geodesic balls $B_{2 r}\left(x_{i}\right), i=1, \ldots, N(r)$, such that the balls $B_{r}\left(x_{i}\right), i=1, \ldots, N(r)$ are disjoint.

The iterated Brezis-Lieb lemma. The following proposition evaluates the $L^{q}$ norms of sequences given by sums of terms with asymptotically disjoint supports. Although it and similar results have appeared elsewhere in literature, for the reader's convenience, we explicitly recall its proof, which is an easy corollary of the well known Brezis-Lieb lemma 5.2. cf. [1].

Lemma 5.2. Let $M$ be a Riemannian manifold, and let $\mu$ be the Riemann measure on $M$. Suppose that $1 \leq q<\infty$. Let $\eta_{k}^{(n)} \in I(M)$ be such that whenever $m \neq n$, the sequence $k \mapsto \eta_{k}^{(m)} \eta_{k}^{(n)^{-1}}$ is discrete. Let $u_{k} \in L^{q}(M)$ be a bounded sequence such that, for each $n \in \mathbb{N}$, the sequence $u_{k} \circ \eta_{k}^{(n)^{-1}}$ converges weakly and almost everywhere to a function which we will denote by $w^{(n)}$. Then, for every $J \in \mathbb{N}$,

$$
\int_{M}\left|u_{k}\right|^{q} d \mu-\sum_{n=1}^{J} \int_{M}\left|w^{(n)}\right|{ }^{q} d \mu-\int_{M}\left|u_{k}-\sum_{n=1}^{J} w^{(n)} \circ \eta_{k}^{(n)}\right|^{q} \rightarrow 0 .
$$

Proof. We use induction. For $J=1$, the statement is immediate from the Brezis-Lieb lemma for the sequence $u_{k} \circ \eta_{k}^{(1)^{-1}}$ whose weak and a.e. limit is $w^{(1)}$. Assume (5.1) is true for $J=\mathrm{m}$, and let us show that it is true for $J=m+1$. Let

$$
\left.v_{k}^{(m)}=u_{k}-\sum_{n=1}^{m} w^{(n)} \circ \eta_{k}^{(n)}\right)
$$

Applying the Brezis-Lieb lemma to the sequence $v_{k}^{(m)} \circ \eta_{k}^{(m+1)^{-1}}$ whose weak and a.e. limit is $w^{(m+1)}$, we obtain from (5.1) the following:

$$
\begin{aligned}
0 & =\lim \left[\int_{M}\left|u_{k}\right|^{p}-\sum_{n=1}^{m} \int_{M}\left|w^{(n)}\right|^{p}-\int_{M}\left|v_{k}^{(m)} \circ \eta_{k}^{(n)}(-1)\right|^{p}\right] \\
& =\lim \left[\int_{M}\left|u_{k}\right|^{p}-\sum_{n=1}^{m} \int_{M}\left|w^{(n)}\right|^{p}-\int_{M}\left|w^{(m+1)}\right|^{p}-\int_{M}\left|v_{k}^{(m+1)} \circ \eta_{k}^{(n)}(-1)\right|^{p}\right],
\end{aligned}
$$

which immediately gives (5.1) for $J=m+1$. 


\section{References}

[1] H. BrÉzis ANd E. Lieb, A relation between pointwise convergence of functions and convergence of functionals, Proc. Amer. Math. Soc. 88 (1983), 486-490.

[2] S. Coleman, V. Glazer, and A. Martin, Action minima among solutions to a class of euclidean scalar field equations, Comm. in Math. Physics 58 (1978), 211-221.

[3] M. Cwikel and K. Tintarev, Rev. Mat. Complut. (online first) doi:10.1007/ s13163-011-0087-2.

[4] M.P. Do Carmo, Riemannian Geometry, Birkhauser, Boston, Basel, Berlin, 1992.

[5] E. Hebey, Nonlinear analysis on manifolds: Sobolev spaces and inequalities, AMS, Providence, Rhode Island 1999.

[6] E. Hebey and M. Vaugon, Sobolev spaces in the presence of symmetries, J. Math. Pures. Appl. 76 (1997), 34-70.

[7] E. Lieb, On the lowest eigenvalue of the Laplacian for the intersection of two domains, Invent. Math. 74 (1983), 441-448.

[8] P.-L. Lions, Symétrie et compacité dans les espaces de Sobolev, J. Funct. Anal. 49 (1982), 315-334.

[9] P.-L. Lions, The concentration-compactness principle in the calculus of variations. The locally compact case, part 1, Ann. Inst. H. Poincare, Analyse non linéaire 1 (1984), 109-1453.

[10] I. Schindler And K. Tintarev, An abstract version of the concentration compactness principle. Revista Matematica Complutense, 15 (2002), 417-436.

[11] W. Sickel And L. Skrzypczak, Radial subspaces of Besov and LizorkinTriebel classes: extended Strauss lemma and compactness of embeddings, J. Fourier Analysus Appl. 6 (2000), 639-662.

[12] L. Skrzypczak, Rotation invariant subspaces of Besov and Tribel-Lizorkin space: compactness of embeddings, smoothness and decay of functions, Revista Mat. Iberoamericana 18 (2002), 267-299.

[13] L. Skrzypczak, Heat extensions, optimal atomic decompositions and Sobolev embeddings in presence of symmetries on manifolds, Math. Z. 243 (2003), 245773 .

[14] W. A. Strauss, Existence of solitary waves in higher dimensions, Comm. in Math. Physics 55 (1977), 149-162.

[15] R. S. Strichartz, Analysis of the Laplacian on a complete Riemannian manifold, J. Func. Anal. 52 (1983), 48-79.

[16] T. TAO, A pseudoconformal compactification of the nonlinear Schrödinger equation and applications, New York J. Math. 15 (2009), 265-282.

[17] K. Tintarev and K.-H. Fieseler, Concentration-compactness: functionalanalytic grounds and applications, Imperial College Press 2007. 
LESZEK SkRZypCZAK

Faculty of Mathematics and Computer Science,

Adam Mickiewicz University,

ul. Umultowska 87,

61-879 Poznan,

Poland

e-mail: 1skrzyp@amu.edu.pl

Cyril Tintarev

Uppsala University,

P.O. Box 480,

SE-751 06 Uppsala,

Sweden

e-mail: kyril.tintarev@math.uu.se

Received: 27 December 2012 